\title{
Características sociodemográficas, económicas, ocupacionales y de percepción de salud que explican la disfuncionalidad familiar de trabajadores informales "vendedores" del centro de Medellín
}

\author{
Sociodemographic, economic, occupational and health perception characteristics which explain the \\ dysfunctional family of informal "sellers" who work of the center of Medellín
}

\section{María Osley Garzón-Duque 1*, María Doris Cardona-Arango², Fabio León Rodríguez-Ospina², Ángela María Segura- \\ Cardona 4}

1 Magister en Epidemiología, estudiante Doctorado Epidemiología y Bioestadística. Universidad CES. Medellín, Colombia. email:mgarzon@ces.edu.co

2 Doctora en Demografía. Universidad CES. Medellín, Colombia. e-mail: dcardona@ces.edu.co

3 Magister en Salud Pública, estudiante Doctorado en Demografía. Facultad Nacional de Salud Pública, Universidad de Antioquia. Medellín, Colombia. e-mail: fabiorodriguez@udea.edu.co

4 Doctora en Epidemiología. Universidad CES. Medellín, Colombia. e-mail: asegura@ces.edu.co económicas, ocupacionales y de percepción de salud que explican la disfuncionalidad familiar de trabajadores informales "vendedores" del centro de Medellín. Univ. Salud. 2016;18(3):447-461. DOI: http://dx.doi.org/10.22267/rus.161803.50

\section{Resumen}

Objetivo: Identificar características sociodemográficas, económicas, ocupacionales y de percepción de salud que expliquen la disfuncionalidad familiar de trabajadores informales "vendedores" del centro de Medellín. Materiales y métodos: Estudio descriptivo transversal con fuentes primarias de información, muestra de 170 trabajadores en el año 2009 con encuesta que incluía el SF-36 y el APGAR familiar. Resultados: El 35\% presentó riesgo de disfunción familiar leve o severa, siendo menor la prevalencia para quienes no eran cabeza de familia (RP: 0,59. IC95\%:0,38;94) y vendían bebidas-picadura y dulce (RP:0,29. IC-95\%: 0,11;0,77), fue mayor para quienes percibían menos ingresos mes (Me: \$300.000; Rq: 242,000), tenían menor número de personas a su cargo (Me:2; Rq: 2) y eran del estrato socioeconómico bajo (RP:2,82. IC-95\%:1,40;5,67). Se observaron diferencias para siete de los ocho dominios del SF36. Conclusiones: Factores laborales y extra laborales en esta población trabajadora aportan a la explicación de la prevalencia de riesgo de disfuncionalidad familiar, ayudando a configurar su condición de vulnerabilidad laboral.

Palabras clave: Trabajadores informales; trabajo; epidemiología. (Fuente: DeCS, Bireme).

\begin{abstract}
Objective: Identify socio-demographic, economic, occupational and health perception characteristics which explain the dysfunctional family of informal "sellers" who work in the center of Medellín. Materials and methods: A descriptive observational cross-sectional study was performed using primary sources of information to a sample of 170 workers in the year 2009 with a survey that included the SF-36 and the family APGAR. Results: The 35\% of those polled presented risk of mild or severe dysfunction family, being less the prevalence for those who were not householders (RP: 0.59.) CI95\%: 0,38; 94) and sold drinks - bite and sweet (RP: 0,29. CI-95\%: 0.11; 0.77), it was
\end{abstract}


greater for those who perceived less income in the month (Me: \$300,000; RQ: 242,000), had fewer dependants (Me: 2; Rq: 2) and belonged to a low socio-economic strata (RP: 2, 82. CI - 95\%:1, 40; 5.67). The differences for seven of the eight domains of the SF-36 were observed. Conclusions: The labor and extra labor factors in this working population provide information to the explanation of the prevalence of risk of dysfunctional family, helping to configure their condition of labor vulnerability.

Keywords: Informal workers, work, epidemiology. (Source: DeCS, Bireme).

\section{Introducción}

Los empleos de subsistencia para quienes laboran en las calles y aceras de la ciudad de Medellín son un tema de importancia en salud pública, que hace necesario el estudio de la problemática, incluyendo aspectos que exploren sus condiciones demográficas, económicas, ocupacionales de percepción de salud y cómo estas condiciones pueden afectar su funcionalidad familiar, siendo este un aspecto que va más allá de su labor en los puestos de venta, con el potencial de afectarlos a nivel personal, social y familiar, estas son algunas de las razones para que en el presente estudio, el evento de interés sea funcionalidad familiar autoreportada por un grupo de trabajadores.

Gallego ${ }^{1}$, refiere que es difícil definir la familia, sin embargo, esta ha sido, es y seguirá siendo el lugar en el que una asociación de personas comparten sus propósitos de vida para tratar de mantenerse unidos en el tiempo ${ }^{2}$. Minuchin ${ }^{3}$ la entiende como un sistema que se transforma a partir de la influencia de los elementos externos que la rodean y que pueden modificar su dinámica interna. Dentro de éstos elementos están las condiciones sociodemográficas, económicas, el trabajo y las condiciones en las que este se realiza, las cuales facilitan o dificultan mejores o peores condiciones de vida y de salud de un núcleo familiar, razón por la cual, es de especial interés explorar la funcionalidad familiar en trabajadores que tienen empleos de subsistencia $\mathrm{y} / \mathrm{o}$ precarios $^{4-7}$, tal como lo describe la Comisión sobre Determinantes Sociales de la Salud (CDSS) ${ }^{8}$, investigaciones y reportes oficiales $2,8-12$, la forma de enfermar y morir de las personas está determinada en gran parte por las condiciones sociales en las que viven y trabajan.
Otro elemento de especial interés, tiene que ver con la necesidad de aportar elementos a un argumento que puede ser aplicado a la población de trabajadores informales venteros del centro de Medellín, el cual indica en términos generales, que la población en condiciones de pobreza puede presentar, a su vez, situaciones difíciles a nivel familiar y laboral ${ }^{11,12}$, y si el trabajo es la fuerza que puede potenciar o desvirtuar muchos de los logros y frustraciones familiares, es preciso observar la funcionalidad familiar de quienes ejercen empleos de subsistencia en diferentes momentos del tiempo, con el fin de tener herramientas comparativas para un tema escasamente explorado que requiere un análisis permanente. El presente estudio buscó identificar las características sociodemográficas, económicas, ocupacionales, de la vivienda y percepción de salud asociadas a la disfuncionalidad familiar de un grupo de trabajadores informales "vendedores" del centro de Medellín, con el propósito de hacer más visible algunos de los factores laborales y extra laborales que podrían estar afectando su vida familiar y laboral, y aportando a la configuración de su vulnerabilidad laboral.

\section{Materiales y métodos}

Estudio exploratorio - transversal con fuentes primarias de información de las condiciones de salud enfermedad de un grupo de trabajadores informales "vendedores" del centro de Medellín 2008-200913 en el que se exploró la percepción de disfuncionalidad familiar, tema del que se derivó la presente pregunta de investigación.

La población fue constituida por un censo realizado en el año 2008 a 423 vendedores en sus puestos de venta. De ésta, se tomó una muestra estadísticamente representativa con selección 
aleatoria de 170 trabajadores, a los que se les aplicó una encuesta asistida en su puesto de venta entre enero y abril del año 2009. El estudio y todas sus actividades fueron concertados con los representantes de los trabajadores y los trabajadores. Se incluyeron quienes contaban con permiso para vender sus productos en las calles y aceras de la ciudad, los mayores de 18 años, que pertenecían a alguna asociación de venteros y tenían mínimo cinco años en su oficio. Fueron excluidos quienes abandonaron su labor por más de seis meses y quienes cambiaron la ubicación de sus puestos de venta. El 4,5\% de la no respuesta se debió a que el trabajador ya no cumplía con los criterios de inclusión al momento de encuesta o por motivos de orden público en el centro de la ciudad.

Se tomaron datos para variables sociodemográficas, económicas, ocupacionales, de la vivienda y de salud, consideradas como explicativas o independientes. La percepción de salud física y psicológica se estudió mediante la aplicación del cuestionario genérico SF-36 adaptado transculturalmente para Colombia por García y Lugo ${ }^{14}$. El cuestionario admite puntajes entre cero (pésimo) y cien (óptimo) para cada uno de los ocho dominios y el cambio de salud en el último año. La prevalencia de riesgo de disfuncionalidad familiar fue tomada como la variable dependiente e identificada con el APGAR familiar, instrumento propuesto por Smilkstein en $1978^{15}$ que evalúa adaptabilidad, participación, ganancia, afecto y recursos; cada ítem se valora entre 0 y 4 , para un puntaje máximo de 20 , ha sido sometido a diferentes procesos de validación ${ }^{16,17}$ y se ha utilizado para el trabajo con diferentes grupos poblacionales ${ }^{18-21}$.

Se realizó análisis descriptivo, y con el análisis bivariado se exploraron asociaciones no causales entre las variables consideradas como explicativas (sociodemográficas, económicas, ocupacionales, de la vivienda y de salud) y la percepción de disfuncionalidad familiar (como dependiente), recategorizada en: leve de 10 a 13 puntos, severa de 0 a 9 puntos - (Si), y normal de 14 a 20 puntos - (No). También se recategorizó la percepción de salud en: mala (00 a 40), regular ( 41 a 60 ), buena ( 61 a 80 ) y muy buena ( 81 a 100 ).
Se realizaron pruebas de asociación $\mathrm{Chi}^{2}$ de Pearson o Fisher y cálculo de la RP con sus IC:95\%, se verificó la distribución de los datos cuantitativos y se calcularon diferencias de medianas (U de Mann-Withney) y una diferencia de promedios para la edad (t-student).

El análisis multivariado se realizó con Regresión logística explicativa para identificar los factores que más aportarán a la explicación de la disfuncionalidad familiar de este grupo de trabajadores. Los trabajadores se agruparon según los productos que vendían de la siguiente forma: productos de cosecha y perecederos, bebidas picadura y dulce, comidas rápidas, mercancía y cacharro; siendo esta última la categoría de referencia para las comparaciones. Todas las pruebas se trabajaron con una confianza del $95 \%$ y un error del 5\%. Los cálculos fueron realizados en el programa SPSS ${ }^{\circledR}$, versión 21 y la diagramación de las tablas fue en el programa Excel.

\section{Consideraciones éticas}

El estudio fue aprobado y clasificado como de riesgo mínimo por el Comité de Bioética de la Facultad Nacional de Salud Pública - Universidad de Antioquia. Cada "ventero" dio su consentimiento informado por escrito, tal como lo estipulan las normas de ética vigentes para el país (Resolución 008430 de 1994) y el Código de Helsinki.

\section{Resultados}

La edad promedio de la población en estudio fue de 45 años (DE $\pm 11,85$ años), siendo los 37 años la edad más frecuente, $64,7 \%$ (110) de los trabajadores eran hombres. El 50\% tenía cinco años de escolaridad o menos (Rq: 3,1), el 93,5\% (159) de los trabajadores estaba afiliado a salud, $81,2 \%$ (124) era del régimen subsidiado, 12,4\% como beneficiarios en el régimen contributivo y el $6,5 \%$ restante eran vinculados. Para el $50 \%$ de ellos, el gasto de bolsillo por recibir atención en salud fue de \$68,564 (+/-: 179,940). La mitad percibían unos ingresos mes de $\$ 400,000$ (Rq: $242,000)$ o menos, sólo el $1,8 \%$ cotizaba a pensiones y el 87,7\% (149) vivían en el estrato socio económico bajo-bajo y bajo. 
El estado civil más frecuente fue la unión libre $32,4 \%$ (55) y casado $24,7 \%$ (42). El 88,2\% (150) reportó ser cabeza de familia, y $50 \%$ de los trabajadores tenían tres personas o menos con dependencia económica. El 24,1\% (41) refirió convivir con otras familias, el tipo de vivienda más comúnmente habitada por los trabajadores fue la casa 60,6\% (103), y el apartamento 24,7\% (42) respectivamente, sin embargo, un 10\% (17) de ellos manifestó vivir en un cuarto. El 45,9\% de los trabajadores pagaba arriendo, y 41,2\% (70) refirió que la vivienda era propia, y alrededor del $50 \%$ manifestó que el estado físico de la misma era regular, para el 38,8\% (66) era bueno y el $12,4 \%$ (21) lo calificaba como malo.

Se observó una población con estabilidad en su oficio, reflejada en su antigüedad ( $\mathrm{Me}=18,5$ : Rq: 15,0 ) y el $50 \%$ de los trabajadores, tenían 7 años o menos de laborar en el mismo sector de venta. En promedio trabajaban seis días a la semana (DE $\pm 0,5)$ durante 10,5 (DE $\pm 2,1)$ horas diarias. Para el 70,0\% (119) de ellos, su puesto de venta estaba en el espacio público sólo para su jornada laboral (semiestacionario), el puesto de venta del 25,3\% (43) permanecía en el espacio público aun después de su jornada laboral (estacionario), y el $4,7 \%$ (8) tenía puestos ambulantes. El 56,5\% (96) de ellos vendía mercancía y cacharro, el 27,6\% (47) vendía productos de cosecha y perecederos. En menor proporción vendían bebidas - picadura y dulce 12,4\% (21), y comidas rápidas el 3,5\% (6). Para el 22,9\% (39) de los trabajadores, el único oficio que habían desempeñado en su vida era el de vendedor y el 20,6\% (35) refirió que había sido obrero, antes que vendedor $18,8 \%$ (32).

\section{Percepción de salud física y psicológica}

Se observó una percepción de salud física y mental aceptable para esta población, sin embargo, hubo dominios que presentaron más bajos puntajes como cambio de salud en el último año $(\mathrm{Me}=60: \mathrm{Rq}: 20)$, salud general $(\mathrm{Me}=65: \mathrm{Rq}$ : 30) y desempeño emocional ( $\mathrm{Me}=66: \mathrm{Rq}: 100)$.

\section{Percepción de funcionalidad familiar}

El 50\% de los trabajadores presentó un puntaje de funcionalidad familiar de 18 (Rq: 11,25) o menos, siendo 20 el puntaje más frecuente. El $10 \%$ de los trabajadores encuestados presentaron una disfunción familiar leve y el $25,3 \%$ perciben que la disfunción de sus familias es severa; es decir, no les satisface la ayuda que reciben, la participación que les brindan y les permiten, la aceptación y apoyo de sus deseos para emprender nuevas actividades, como les expresan afecto y responden a sus emociones, y no les satisface como comparten con sus familias.

\section{Prevalencia de riesgo de disfuncionalidad familiar, según características sociodemográficas, de la vivienda y laborales} No se observaron diferencias estadísticamente significativas, entre los promedios de edad de los trabajadores que presentaron algún tipo de disfuncionalidad familiar y aquellos que no la presentaron $(p=0,11)$; sin embargo, se observa una tendencia de mayor promedio de edad $(47,3$ años) para aquellos que reportaron prevalencia de disfuncionalidad familiar $(\mathrm{t}=3,26$ : IC; - 0,74 ; $7,26)$, con respecto a quienes no la presentaron (44,1 años).

No se presentaron diferencias estadísticamente significativas entre los años de escolaridad y gastos de bolsillo por atención en salud el mes anterior a la encuesta; sin embargo, el 50\% de los trabajadores con riesgo de disfuncionalidad familiar tenían cuatro años o menos de escolaridad que quienes no presentaron esta prevalencia de disfuncionalidad, teniendo presente que los años de escolaridad, eran cinco o menos (Tabla 1).

De otro lado, pudo observarse que quienes presentaban prevalencia de riesgo de disfuncionalidad familiar tenían menos ingresos al mes y menos personas a su cargo, siendo estas diferencias estadísticamente significativas $(\mathrm{p}=$ 0,00 ) (Tabla 2).

No se observaron asociaciones estadísticamente significativas que indiquen que la prevalencia de riesgo de disfuncionalidad familiar sea mayor o menor al comparar grupos según sexo, estado civil, pago de pensión, convivir con otras familias o estar afiliado al sistema de salud. De otro lado, pudo observarse que los trabajadores que no eran cabeza de familia, tenían $41 \%$ menos prevalencia de riesgo de disfuncionalidad familiar que 
aquellos que si lo eran, y esta menor prevalencia podría estar entre el $6 \%$ y $62 \%$ respectivamente (RP; 0,59: IC 95\%: 0,38; 94) ( $p=0,04)$. No se observaron asociaciones que indicaran que la prevalencia de riesgo fuera mayor o menor para alguna de las categorías del estado civil (Tabla 3). Se presentaron diferencias estadísticamente significativas $(p=0,04)$ entre el estrato socioeconómico bajo-bajo y el bajo, indicando que por cada trabajador del estrato bajo-bajo que manifestó tener disfuncionalidad familiar, se presentaron 2,8 trabajadores del estrato bajo que tenían esta misma prevalencia de riesgo (RP: 2,82 IC: 1,$4 ; 5,7)$ (Tabla 3).

Tabla 1. Percepción de la disfuncionalidad familiar en los trabajadores informales "venteros" del centro de Medellín según variables sociodemográficas e indicadores sintéticos de exposición laboral, 2008-2009

\begin{tabular}{|c|c|c|c|c|c|}
\hline \multirow{3}{*}{$\begin{array}{l}\text { Característica sociodemográfica, } \\
\text { laboral }\end{array}$} & \multicolumn{2}{|c|}{ Disfunción Familiar } & \multicolumn{2}{|c|}{ Mediana (Rq) } & \multirow{3}{*}{$\begin{array}{l}\text { Prueba U-MW } \\
\text { (Valor p)* }\end{array}$} \\
\hline & $\mathbf{S i}$ & No & $\mathbf{S i}$ & No & \\
\hline & n (\%) & n (\%) & & & \\
\hline Escolaridad & $60(35,3)$ & $110(64,7)$ & $4(17)$ & $5(12)$ & $2803(0,09)$ \\
\hline Pago salud & $60(35,3)$ & $110(64,7)$ & $0(180,000)$ & $0(75,000)$ & $3147,5(0,57)$ \\
\hline Ingresos mes & $60(35,3)$ & $110(64,7)$ & $300000(800,000)$ & $400000(800,000)$ & $2445(0,00)$ \\
\hline Personas a cargo del trabajador & $60(35,3)$ & $110(64,7)$ & $2(5)$ & $3(9)$ & $2611(0,02)$ \\
\hline Horas de trabajo al día & $60(35,3)$ & $110(64,7)$ & $10(12)$ & $10(12)$ & $3057(0,42)$ \\
\hline Días de trabajo a la semana & $60(35,3)$ & $110(64,7)$ & $6(2)$ & $6(2)$ & $2858(0,08)$ \\
\hline Antigüedad en el oficio & $60(35,3)$ & $110(64,7)$ & $18(36)$ & $18(46)$ & $2858(0,15)$ \\
\hline Antigüedad en el sector & $60(35,3)$ & $110(64,7)$ & $8(27)$ & $6(27)$ & $3160(0,57)$ \\
\hline
\end{tabular}

Tabla 2. Percepción de disfuncionalidad familiar según características sociodemográficas y familiares de los trabajadores informales "venteros" del centro de Medellín, 2008-2009

\begin{tabular}{|c|c|c|c|c|c|c|}
\hline \multirow{2}{*}{\multicolumn{2}{|c|}{ Característica sociodemográfica }} & \multicolumn{2}{|c|}{ Disfunción Familiar APGAR } & \multirow{2}{*}{ Total fila } & \multirow{2}{*}{ RP (IC : 95\%) } & \multirow[b]{2}{*}{ Chi2 (Valor p) } \\
\hline & & Si $(n=60)$ & No $(n=110)$ & & & \\
\hline \multirow{2}{*}{ Sexo* } & Hombre & 34 & 76 & 110 & \multirow{2}{*}{$0,71(0,47 ; 1,06)$} & \multirow{2}{*}{$2,62(0,10)$} \\
\hline & Mujer & 26 & 34 & 60 & & \\
\hline \multirow{2}{*}{ Pensión** } & No & 58 & 109 & 167 & \multirow{2}{*}{$1,96(0,39 ; 9,74)$} & \multirow{2}{*}{$1,31(0,28)$} \\
\hline & $\mathrm{Si}$ & 2 & 1 & 3 & & \\
\hline \multirow{2}{*}{ Cabeza familia* } & $\mathrm{Si}$ & 49 & 101 & 150 & \multirow{2}{*}{$0,59(0,38 ; 94)$} & \multirow{2}{*}{$3,85(0,04)$} \\
\hline & No & 11 & 9 & 20 & & \\
\hline \multirow{2}{*}{ Convive con otras familias* } & $\mathrm{Si}$ & 19 & 22 & 41 & \multirow{2}{*}{$1,46(0,96 ; 2,21)$} & \multirow{2}{*}{$2,88(0,08)$} \\
\hline & No & 41 & 88 & 129 & & \\
\hline \multirow{2}{*}{ Afiliación Salud** } & No & 6 & 5 & 11 & \multirow{2}{*}{$1,60(0,89 ; 2,87)$} & \multirow{2}{*}{$1,89(0,09)$} \\
\hline & $\mathrm{Si}$ & 54 & 105 & 159 & & \\
\hline
\end{tabular}

*Prueba chi ${ }^{2}$ de Pearson

** Prueba chi $^{2}$ de Fisher

Para el tipo de vivienda, tenencia de la vivienda y estado de la vivienda que habitaban los trabajadores al momento de la encuesta, no se observaron asociaciones estadísticamente significativas que indicarán una mayor o menor prevalencia de riesgo de disfuncionalidad familiar en las categorías de éstas variables comparadas (Tabla 3). 
Tabla 3. Características laborales y familiares del trabajador informal "ventero" del centro de Medellín exploradas, según percepción de funcionalidad familiar, 2008-2009

\begin{tabular}{|c|c|c|c|c|c|}
\hline \multirow{2}{*}{ Característica o condición } & \multicolumn{2}{|c|}{ Disfunción Familiar APGAR } & \multirow{2}{*}{$\begin{array}{c}\text { Total } \\
\text { fila }\end{array}$} & \multirow{2}{*}{ RP (IC : 95\%) } & \multirow{2}{*}{$\begin{array}{c}\text { Chi2 (Valor } \\
\text { p) }\end{array}$} \\
\hline & $\operatorname{Si}(n=60)$ & No $(n=110)$ & & & \\
\hline \multicolumn{6}{|l|}{ Estado civil } \\
\hline Separado & 18 & 23 & 41 & 1,0 & $7,24(0,11)$ \\
\hline Casado & 11 & 31 & 42 & $1,18(0,43 ; 3,20)$ & $0,10(0,75)$ \\
\hline Viudo & 4 & 3 & 7 & $2,60(0,91 ; 7,38)$ & $3,22(0,07)$ \\
\hline Unión libre & 15 & 40 & 55 & $0,69(0,13 ; 3,75)$ & $0,18(0,67)$ \\
\hline Soltero & 12 & 13 & 25 & $2,46(0,92 ; 6,58)$ & $3,22(0,07)$ \\
\hline \multicolumn{6}{|c|}{ Estrato socio-económico de la vivienda que habitaba al momento del estudio } \\
\hline Bajo-Bajo & 33 & 37 & 70 & 1,0 & $8,64(0,03)$ \\
\hline Bajo & 19 & 60 & 79 & $2,82(1,40 ; 5,67)$ & $8,47(0,04)$ \\
\hline Bajo -medio & 7 & 12 & 19 & $1,53(0,54 ; 4,34)$ & $0,64(0,42)$ \\
\hline Medio & 1 & 1 & 2 & $0,89(0,05 ; 14,83)$ & $0,006(0,94)$ \\
\hline \multicolumn{6}{|c|}{ Tipo de vivienda que habitaba el trabajador } \\
\hline Casa & 2 & 4 & 6 & 1,0 & $2,24(0,69)$ \\
\hline Apartamento & 11 & 31 & 42 & $1,72(0,77 ; 3,80)$ & $1,78(0,18)$ \\
\hline Cuarto & 7 & 10 & 17 & $0,87(0,31 ; 2,41)$ & $0,68(0,79)$ \\
\hline Inquilinato & 39 & 64 & 103 & $0,61(0,37 ; 10,02)$ & $0,12(0,73)$ \\
\hline Otro & 1 & 1 & 2 & $1,22(0,21 ; 6,96)$ & $0,05(0,82)$ \\
\hline \multicolumn{6}{|l|}{ Tenencia de la vivienda } \\
\hline Propia & 30 & 40 & 70 & 1,0 & $2,99(0,22)$ \\
\hline Otro & 7 & 15 & 22 & $0,56(0,28 ; 1,09)$ & $2,84(0,09)$ \\
\hline Arrendada & 23 & 55 & 78 & $0,89(0,32 ; 2,49)$ & $0,04(0,83)$ \\
\hline \multicolumn{6}{|l|}{ Estado de la vivienda que habitaba } \\
\hline Malo & 20 & 46 & 66 & 1,0 & $2,10(0,35)$ \\
\hline Regular & 30 & 53 & 83 & $1,61(0,61 ; 4,22)$ & $0,92(0,34)$ \\
\hline Bueno & 10 & 11 & 21 & $2,09(0,76 ; 5,71)$ & $2,07(0,15)$ \\
\hline \multicolumn{6}{|l|}{ Tipo de venta } \\
\hline Ambulante & 2 & 6 & 8 & 1,0 & $1,17(0,55)$ \\
\hline Semi-estacionario & 45 & 74 & 119 & $0,55(0,11 ; 2,83)$ & $0,51(0,47)$ \\
\hline Estacionario & 13 & 30 & 43 & $0,7(0,14 ; 4,33)$ & $0,89(0,77)$ \\
\hline \multicolumn{6}{|l|}{ Tipología de venta } \\
\hline Mercancía y cacharro & 27 & 69 & 96 & 1,0 & $6,79(0,07)$ \\
\hline Productos de cosecha y perecederos & 19 & 28 & 47 & $0,58(0,28 ; 1,20)$ & $2,16(0,14)$ \\
\hline Bebidas - picadura y dulce & 12 & 9 & 21 & $0,29(0,11 ; 0,77)$ & $6,11(0,01)$ \\
\hline Comidas rápidas & 2 & 4 & 6 & $0,78(0,13 ; 4,52)$ & $0,07(0,78)$ \\
\hline \multicolumn{6}{|c|}{ Oficio que desempeñaba antes de ser ventero } \\
\hline Agricultor & 11 & 20 & 31 & 1,0 & $5,32(0,38)$ \\
\hline Obrero & 7 & 28 & 35 & $2,20(0,73 ; 6,66)$ & $1,94(0,16)$ \\
\hline Empleado & 15 & 17 & 32 & $0,62(0,23 ; 1,71)$ & $0,84(0,36)$ \\
\hline Desempleado & 1 & 0 & 1 & $0,00(0,00 ;-)$ & $0,00(1,0)$ \\
\hline Ninguno & 14 & 25 & 39 & $0,98(0,37 ; 2,63)$ & $0,01(0,97)$ \\
\hline Otro & 12 & 20 & 32 & $0,92(0,33 ; 2,56)$ & $0,03(0,83)$ \\
\hline
\end{tabular}

*Prueba $\mathrm{chi}^{2}$ de Pearson 
Prevalencia de riesgo de disfuncionalidad familiar según indicadores sintéticos de exposición laboral

Para las horas de trabajo al día, días de trabajo a la semana, antigüedad en el oficio de vendedor informal, y antigüedad en el sector de venta, no se observaron diferencias que indiquen una mayor o menor prevalencia de disfuncionalidad familiar para alguna de estas variables exploradas (Tabla $1)$.

No se observaron asociaciones según el tipo de venta $y$ el oficio que desempeñaban los trabajadores antes de ser "venteros" que indiquen que la prevalencia de riesgo de disfuncionalidad familiar sea mayor o menor para algún tipo de venta en particular o para algún oficio antes de ser ventero (Tabla 3).

Se observaron asociaciones estadísticamente significativas $(p=0,01)$ entre los vendedores de mercancía y cacharro, y los de bebidas-picadura y dulce, indicando que para los vendedores de picadura y dulce (cigarrilos, confites etc.) la prevalencia de riesgo de disfuncionalidad familiar era 2,41 veces mayor que para aquellos que vendían mercancía y cacharro (RP: 3,41 IC: 1,29 ; 9,01) (Tabla 3).
Prevalencia de riesgo de disfuncionalidad familiar según percepción de salud física y psicológica (SF-36)

Se observó que las medianas para 7 de los 8 dominios, y el cambio de salud en el último año eran de 60 puntos y más, para quienes reportaron riesgo de disfuncionalidad familiar. Sin embargo, se observó que la mitad de los trabajadores con riesgo de disfuncionalidad familiar, presentaron un puntaje de 0,0 para el dominio de desempeño emocional, con diferencias estadísticamente significativas $(p=0,00)$ (Figura 1).

Los puntajes de las medianas para los dominios de dolor corporal, función social, salud general, salud mental $y$ vitalidad, fueron significativamente menores $(p=0,00)$ para aquellos trabajadores que reportaron mayores prevalencias de riesgo de disfuncionalidad familiar (leve o severa).

El dominio de función física no se relacionó significativamente con la prevalencia de riesgo de disfuncionalidad familiar $(\mathrm{p}=0.05)$ si se observaron valores menores en quienes presentaron prevalencia de riesgo de disfuncionalidad familiar (Figura 1).

Figura 1. Disfuncionalidad familiar según percepción de salud física y psicológica (SF-36) para el grupo de trabajadores informales "venteros" en estudio. Medellín 2008-2009

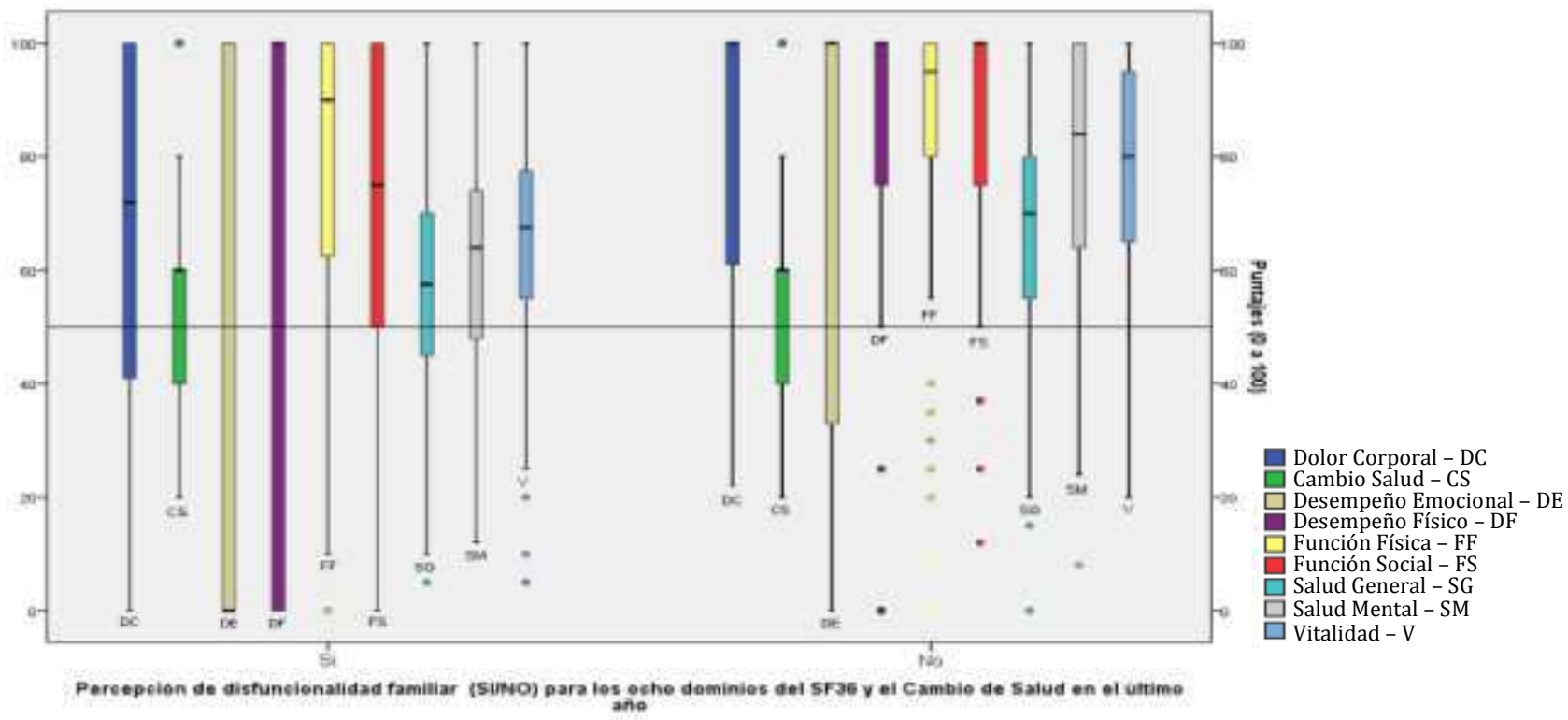


Características sociodemográficas, laborales y de la vivienda que más aportan a la explicación de la prevalencia de disfuncionalidad familiar

Se observó que los trabajadores entre 36 y 50 años tenían menos disfuncionalidad familiar que aquellos mayores de 60 años para el caso del análisis bivariado, pero este factor dejó de perfilarse como protector al ajustar por las demás variables de este grupo. Tener de 20 a 35 años de edad pasó de una tendencia de factor protector a factor de riesgo. De otro lado, estar casado y en unión libre, fueron factores que se perfilaron como protectores de la percepción de disfuncionalidad familiar tanto para el análisis bivariado, como al ajustar por las demás variables sociodemográficas, laborales y de la vivienda. Una tendencia de menor percepción de disfuncionalidad familiar, fue observada en el análisis bivariado, para quienes vivían en casa arrendada, pero en el análisis multivariado esta tenencia se comportó como factor de riesgo, al igual que para la categoría otro tipo de vivienda.

Haber sido vendedor entre 16 a 40 años, tanto en el análisis bivariado como multivariado se comportó como un factor que protege de la percepción de disfunción familiar, al igual que haber sido obrero antes de ser ventero.

Para la tipología de venta, se observaron asociaciones estadísticamente significativas tanto para el análisis bivariado como el multivariado, en ambos casos, aquellos trabajadores que vendían bebidas - picadura y dulce presentaron mayores prevalencias de riesgo de disfuncionalidad familiar que aquellos que vendían mercancía y cacharro, presentándose una mayor capacidad explicativa de esta prevalencia de riesgo en el análisis multivariado, pues el RP ajustado fue mayor que el crudo, indicando que por cada trabajador que vendía mercancía y cacharro, y presentaba riesgo de disfunción familiar se presentaban 3,5 trabajadores que vendían bebidas - picadura y dulce, y percibían disfunción familiar (IC 95\%: 1,07 ; 11,67) (Tabla 4).
Dominios de percepción de salud física y psicológica que más aportan a la explicación de la prevalencia de disfuncionalidad familiar Con relación a estas condiciones se observaron las siguientes características: el desempeño emocional regular (41 a 60 puntos) que se había comportado como un evidente factor de riesgo en el RP crudo (RP: 4.93, IC95\% $2.38 ; 10.20$ ), perdió su poder explicativo al ajustarse por los demás dominios de esta escala, disminuyendo considerablemente su RP y perdiendo la significancia estadística, similar situación se observó en el RP crudo para la percepción de desempeño físico en su categoría malo $(0.0$ a 40 puntos - RP: 1.9, IC95\% 2.29 ; 4.81), función social regular (41 a 60 puntos - RP: 9.28, IC95\% 2.27 ; 37.82), función social mala $(0.0$ a 40 puntos - RP: 5.22 , IC95\% $1.90 ; 14.29)$ y función social buena (61 a 80 puntos - RP: 3.11, IC95\% $1.40 ; 6.94$ ), dominios que siguieron comportándose en el RP ajustado como factores de riesgo, pero perdieron su capacidad explicativa en conjunto, en términos de la significancia estadística.

Esta misma situación se presentó con los Dominios de Vitalidad para sus categorías regular (41 a 60 puntos - RP: 8.00 , IC95\% $2.93 ; 21.85$ ) y buena (61 a 80 puntos - RP: 4.37, IC95\% 1.85 ; 10.35). No obstante, la situación fue diferente a la observada, con la percepción de Desempeño Físico regular (41 a 60 puntos) que había presentado una tendencia a factor protector con el RP crudo (RP: 0,93; IC95\% 0.23 ; 3.81) pero al ajustarse esta categoría del dominio con los demás dominios de la escala, y las demás categorías del dominio paso a comportarse como un factor protector estadísticamente significativo, con poder explicativo para el modelo (RP: 0.14, IC95\% $0.02 ; 0.99$ ). Para el único dominio que se conservaron todas las categorías de comparación (regular, mala y buena) como factores de riesgo estadísticamente significativo tanto en el RP crudo como en el ajustado fue para el de Salud Mental (Tabla 5). 
Tabla 4. Variables sociodemográficas, de la vivienda y laborales que más aportan a la explicación de la disfuncionalidad familiar en el grupo de trabajadores informales

\begin{tabular}{|c|c|c|c|c|c|c|}
\hline \multirow{2}{*}{ Variable } & \multirow{2}{*}{ RP crudo } & \multicolumn{2}{|c|}{ IC; 95\% } & \multirow{2}{*}{ RP ajust. } & \multicolumn{2}{|c|}{ IC; 95\% } \\
\hline & & $\mathbf{L i}$ & Ls & & $\mathbf{L i}$ & Ls \\
\hline Edad (Cr. 61 a 79 años)* & - & & & - & & \\
\hline Edad (20 a 35 años) & 0,36 & 0,11 & 1,15 & 3,33 & 0,85 & 13,03 \\
\hline Edad (36 a 50 años) & 0,21 & 0,07 & 0,61 & 0,95 & 0,27 & 3,38 \\
\hline Edad (51 a 60 años) & 0,34 & 0,11 & 1,07 & 0,55 & 0,20 & 1,49 \\
\hline Mujer $(\mathrm{Cr})^{*}$ & - & & & - & & \\
\hline Hombre & 1,71 & 0,89 & 3,28 & 0,79 & 0,31 & 2,02 \\
\hline Ingresos mes (Cr. 401,000 a 900,000)* & - & & & - & & \\
\hline Ingresos mes $(100,000 \text { a } 400,000)^{*}$ & 1,87 & 0,96 & 3,66 & 0,97 & 0,40 & 2,36 \\
\hline Soltero $\mathrm{Cr}{ }^{*}$ & - & & & - & & \\
\hline Casado & 0,45 & 0,18 & 1,14 & 0,36 & 0,11 & 1,20 \\
\hline Unión libre & 0,48 & 0,20 & 1,13 & 0,81 & 0,29 & 2,21 \\
\hline Viudo & 1,70 & 0,34 & 8,60 & 1,18 & 0,13 & 10,97 \\
\hline Separado & 1,18 & 0,43 & 3,20 & 1,50 & 0,44 & 5,12 \\
\hline Casa propia Cr.* & - & & & - & & \\
\hline Casa arrendada & 0,56 & 0,28 & 1,10 & 2,35 & 0,69 & 7,97 \\
\hline Otro tipo de tenencia de la vivienda & 0,62 & 0,23 & 1,72 & 1,14 & 0,34 & 3,83 \\
\hline Estrato Medio Cr* & - & & & - & & \\
\hline Estrato bajo - bajo & 1,71 & 0,09 & 31,92 & 1,73 & 0,07 & 41,52 \\
\hline Estrato bajo & 1,53 & 0,54 & 4,34 & 1,59 & 0,45 & 5,66 \\
\hline Estrato Medio - bajo & 0,54 & 0,19 & 1,58 & 0,63 & 0,18 & 2,22 \\
\hline Antigüedad en el oficio (cr. 2 a 15 años)* & - & & & - & & \\
\hline Antigüedad en el oficio 16 a 40 años & 0,48 & 0,19 & 1,21 & 0,71 & 0,34 & 1,45 \\
\hline Tipología de venta (Cr. Mercancía y Cacharro)* & - & & & - & & \\
\hline Productos de cosecha y perecederos & 1,73 & 0,83 & 3,61 & 2,22 & 0,88 & 5,60 \\
\hline Bebidas picadura y dulce & 3,41 & 1,29 & 9,01 & 3,52 & 1,07 & 11,57 \\
\hline Comidas rápidas y otros alimentos & 1,28 & 0,22 & 7,39 & 0,50 & 0,05 & 5,09 \\
\hline Oficio antes de ser ventero (Cr. Ninguno)* & - & & & - & & \\
\hline Agricultor & 0,92 & 0,38 & 2,20 & 1,24 & 0,38 & 4,10 \\
\hline Obrero & 0,42 & 0,16 & 1,08 & 0,50 & 0,16 & 1,59 \\
\hline Desempleado & 1,47 & 0,63 & 3,41 & 2,43 & 0,84 & 7,05 \\
\hline
\end{tabular}

Cr: Categoría de referencia 
Tabla 5. Dominios de la percepción de salud física y psicológica (SF-36) que más aportan a la explicación de la disfuncionalidad familiar en el grupo de trabajadores informales

\begin{tabular}{|c|c|c|c|c|c|c|}
\hline \multirow{2}{*}{ Variable } & \multirow{2}{*}{$\begin{array}{c}\text { RP } \\
\text { crudo }\end{array}$} & \multicolumn{2}{|c|}{ IC; 95\% } & \multirow{2}{*}{ RP ajust. } & \multicolumn{2}{|c|}{ IC; 95\% } \\
\hline & & $\mathbf{L i}$ & Ls & & $\mathbf{L i}$ & Ls \\
\hline Cambio de Salud (Cr: 81 a 100 puntos)* & - & & & - & & \\
\hline Cambio de Salud (41 a 60) & 0,63 & 0,16 & 2,41 & 0,47 & 0,09 & 2,42 \\
\hline Cambio de Salud (00 a 40) & 1,09 & 0,27 & 4,34 & 0,71 & 0,12 & 4,14 \\
\hline Cambio de Salud (61 a 80) & 1,00 & 0,22 & 4,47 & 0,99 & 0,16 & 6,06 \\
\hline Desempeño Emocional (Cr: 81 a 100)* & - & & & - & & \\
\hline Desempeño Emocional (41 a 60) & 4,93 & 2,38 & 10,20 & 1,38 & 0,40 & 4,82 \\
\hline Desempeño Emocional (00 a 40) & 2,10 & 0,77 & 5,77 & 1,18 & 0,32 & 4,42 \\
\hline \multicolumn{7}{|l|}{ Desempeño físico (Cr: 81 a 100)* } \\
\hline Desempeño físico (41 a 60) & 0,93 & 0,23 & 3,81 & 0,14 & 0,02 & 0,99 \\
\hline Desempeño físico (00 a 40) & 2,29 & 1,09 & 4,81 & 0,52 & 0,14 & 1,94 \\
\hline Desempeño físico (61 a 80) & 0,54 & 0,11 & 2,69 & 0,13 & 0,01 & 1,15 \\
\hline Función Física (Cr: 81 a 100)* & - & & & - & & \\
\hline Función Física (41 a 60) & 2,13 & 0,71 & 6,37 & 2,44 & 0,49 & 12,06 \\
\hline Función Física (00 a 40) & 2,44 & 0,84 & 7,06 & 1,00 & 0,13 & 7,71 \\
\hline Función Física (61 a 80) & 1,98 & 0,86 & 4,59 & 2,02 & 0,63 & 6,49 \\
\hline Función Social (Cr: 81 a 100)* & - & & & - & & \\
\hline Función Social (41 a 60) & 9,28 & 2,27 & 37,82 & 2,86 & 0,43 & 19,10 \\
\hline Función Social (00 a 40) & 5,22 & 1,90 & 14,29 & 2,61 & 0,47 & 14,52 \\
\hline Función Social (61 a 80) & 3,11 & 1,40 & 6,94 & 1,18 & 0,35 & 3,94 \\
\hline Salud General (Cr: 81 a 100)* & - & & & - & & \\
\hline Salud General (41 a 60) & 1,65 & 0,65 & 4,21 & 0,28 & 0,07 & 1,12 \\
\hline Salud General (00 a 40) & 2,04 & 0,71 & 5,91 & 0,43 & 0,08 & 2,32 \\
\hline Salud General (61 a 80) & 0,77 & 0,30 & 1,96 & 0,35 & 0,10 & 1,25 \\
\hline Salud Mental (Cr: 81 a 100)* & - & & & - & & \\
\hline Salud Mental (41 a 60) & 7,24 & 2,82 & 18,60 & 5,51 & 1,11 & 27,38 \\
\hline Salud Mental (00 a 40) & 10,17 & 3,02 & 34,20 & 29,40 & 3,20 & 270,14 \\
\hline Salud Mental (61 a 80) & 4,74 & 1,97 & 11,42 & 4,42 & 1,20 & 16,26 \\
\hline Vitalidad (Cr: 81 a 100 puntos) & - & & & - & & \\
\hline Vitalidad (41 a 60 puntos) & 8,00 & 2,93 & 21,85 & 3,27 & 0,63 & 16,91 \\
\hline Vitalidad (00 a 40 puntos) & 3,61 & 0,96 & 13,55 & 0,18 & 0,02 & 2,21 \\
\hline Vitalidad (61 a 80 puntos) & 4,37 & 1,85 & 10,35 & 2,72 & 0,83 & 8,89 \\
\hline
\end{tabular}




\section{Discusión}

En la población de trabajadores informales "vendedores" en estudio, predominaron los hombres, con una edad promedio de 45 años y \pm 5 años de escolaridad, situación muy similar a la reportada por el diagnóstico nacional de condiciones de salud y trabajo de las personas ocupadas en el sector informal para Colombia ${ }^{2}$.

Se ha referido que hasta un $82 \%$ de los trabajadores ocupados en el sector informal, perciben menos de un salario mínimo legal mensual vigente ${ }^{22}$, para el presente estudio el porcentaje observado fue de un $50 \%$ de los trabajadores que percibían mensualmente 400 mil pesos o menos, con valores mínimos hasta de 100 mil pesos.

Aunque más del $90 \%$ de los trabajadores manifestó estar afiliado al sistema de salud, esta mayor afiliación era vía régimen subsidiado y el porcentaje de afiliación al régimen contributivo, era para quienes estaban como beneficiarios de sus parientes. La afiliación al régimen de pensiones fue prácticamente inexistente, situación similar a la observada en el diagnóstico nacional de trabajadores del sector informal a $2008^{22}$, y mucho menor a la registrada por el Informe Notas Fiscales del $2011^{23}$ de Colombia, el cual registra que un $10,1 \%$ de los ocupados informales están afiliados a salud y pensiones, de éstos el $84,6 \%$ cotiza a salud y $10,3 \%$ a pensiones, y tienen bajos niveles de educación $(29,6 \%)$, datos similares a los reportados en la Segunda encuesta nacional de condiciones de seguridad y salud en el trabajo, $2013^{24}$.

Los vendedores vivían principalmente en unión libre y matrimonio, eran cabeza de familia y tenían tres personas a su cargo, similar situación a la reportada por el diagnóstico nacional de trabajadores del sector informal ${ }^{24}$, donde más del $60 \%$ eran cabeza de familia y tenían entre 1 y 4 personas a su cargo.

\section{Condiciones laborales}

Un rasgo característico en estos trabajadores es que el oficio de ventero no es temporal o de emergencia, según le registrado con el número de años en la misma labor. El grupo presentó una carga laboral considerable en términos de las horas que trabajan diariamente, los días que laboran a la semana y su antigüedad en el sector de venta, coincidiendo estos resultados, con los registrados por el diagnóstico para personas ocupadas en el sector informal en el año $2008^{22}$.

\section{Características de la vivienda}

Las viviendas de los trabajadores estaban ubicadas en los estratos más bajos ( 1 y 2 ) y alrededor del 50\% de ellos pagaba arriendo, siendo esta cifra menor que la registrada en un informe del Ministerio de la Protección Social para el año $2008^{22}$. La mitad de los trabajadores consideraba que su vivienda estaba en regular estado y uno de cada cuatro refirió que convivía con otra familia en su vivienda. Características que acompañadas de las difíciles condiciones sociodemográficas y económicas, podrían afectar su percepción de funcionalidad familiar.

\section{Percepción de salud física y psicológica}

En términos generales se observó una aceptable percepción de salud, acorde con los resultados obtenidos por García y Lugo ${ }^{14}$ en la adaptación transcultural del SF-36, sin embargo, llama la atención dominios como cambio de salud en el último año, salud general y desempeño emocional que presentaron bajos puntajes promedio, diferentes a lo observado en un estudio realizado en España con 3915 trabajadores de 239 empresas, donde las mujeres tenían mejor estado de salud general y mental que los hombres, a mayor riesgo de la empresa, los hombres también aumentan el riesgo de padecer alteraciones de salud general, función física y función social, y en empresas pequeñas $\quad(<25$ trabajadores $)$ presentaron alteración de su percepción de salud 25 .

\section{Percepción de disfuncionalidad familiar}

Llama la atención que el 10\% de los trabajadores presentó disfunción familiar leve y el 25,3\% percibían que la disfunción de sus familias era severa. Estos resultados difieren de los presentados en otros estudios para poblaciones diferentes, donde la disfuncionalidad familiar leve es mayor ${ }^{18,20,21}$. El alto porcentaje de disfuncionalidad severa para los vendedores 
informales en estudio, puede estar indicando la necesidad de explorar más aspectos familiares y de salud mental en este tipo de población trabajadora, que faciliten el abordaje con poblaciones comparables a la hora de realizar este tipo de análisis.

\section{Prevalencia de riesgo de disfuncionalidad familiar, según características sociodemográficas, de la vivienda y laborales}

Se identificó una tendencia de mayor promedio de edad, para aquellos que presentaron prevalencia de disfuncionalidad familiar; similares resultados a los presentados en un estudio de México con 100 adultos entre 60 y 91 años de clase media, en ellos, un 35\% presentó disfunción moderada y $14 \%$ grave, se asociaron a la disfunción familiar una mayor edad y la baja escolaridad ${ }^{26}$. Estos resultados difieren de los reportados por un estudio en Cuba, donde para los adultos mayores la familia era funcional ${ }^{27}$. Aquellos que presentaron mayor prevalencia de disfuncionalidad familiar, tenían menos ingresos al mes y menos personas a su cargo. En un estudio con adultos mayores en México quienes estaban jubilados tenían mayor disfuncionalidad familiar que quienes eran laboralmente activos ${ }^{26}$, de otro lado, en un estudio realizado en Itagüí Antioquia con familias que asistían a hogares comunitarios se observó que las familias que ingresaron al hogar comunitario por motivos económicos y/o alimentarios presentan menor nivel de funcionalidad familiar ${ }^{20}$.

No se observaron diferencias en la disfuncionalidad familiar según sexo, estado civil, pago de pensión, convivir con otras familias o estar afiliado al sistema de salud, pero si se observó que quienes no eran cabeza de familia tenían menor prevalencia de disfuncionalidad familiar, similar a lo reportado por Giraldo et al. ${ }^{20}$. Aunque para el presente estudio se observaron diferencias en la disfuncionalidad familiar entre el estrato bajo-bajo y el bajo, no es posible dimensionar los resultados, en términos de lo que pueda estar sucediendo para otros grupos de trabajadores que ejercen sus labores en las calles y aceras de las ciudades, pues al momento no se dispone de argumentos comparativos con relación a este aspecto, cuanto los estudios disponibles para la discusión no contemplaron este factor. Igual dificultad comparativa se presentó para las características de la vivienda (tipo de vivienda, tenencia de la vivienda y estado de la misma), pues no es frecuente la inclusión de este tipo de variables en los estudios de funcionalidad familiar y menos para vendedores de las calles y aceras de las ciudades, siendo esta una limitante para el estudio y sus posibilidades comparativas.

\section{Prevalencia de riesgo de disfuncionalidad familiar según características laborales}

No se observaron diferencias entre las categorías de las variables horas de trabajo al día, días de trabajo a la semana, antigüedad en el oficio, antigüedad en el sector de venta, tipo de venta, oficio desempañado antes de ser ventero y la disfuncionalidad familiar, pero si fueron observadas diferencias entre los vendedores de mercancía y cacharro, y los de bebidas - picadura y dulce, siendo mayor la disfuncionalidad familiar para este último grupo de trabajadores.

Dentro de los estudios consultados no se lograró identificar análisis que incluyeran este tipo de variables, sólo se observó en un estudio con adultos mayores de México, que quienes estaban económicamente activos presentaban mejor funcionalidad familiar ${ }^{26}$, situación diferente a la de los vendedores en estudio, que se constituyen en población económicamente activa.

\section{Prevalencia de riesgo de disfuncionalidad familiar según percepción de salud física y psicológica (SF-36)}

Los dominios de dolor corporal, función social, salud general, salud mental y vitalidad se asociaron significativamente con la prevalencia de riesgo de disfuncionalidad familiar leve o severa, mostrando en todos los casos, que eran más bajos los puntajes de percepción de salud, para quienes tenían mayor prevalencia de riesgo de disfuncionalidad familiar, indicando una alta relación entre la percepción de salud física y mental, y la percepción de disfuncionalidad familiar del grupo de trabajadores participantes en el estudio. Aunque no fue una diferencia significativa, la mediana de función física fue peor para quienes presentaron mayor prevalencia de 
disfuncionalidad familiar. Es importante tener presente, que no es posible comparar estos hallazgos a la luz de otros estudios con población similar, pues en la literatura consultada sólo se observaron dos estudios con aplicación del SF-36 para población trabajadora en la economía formal, uno en Cuba y otro en España ${ }^{25-28 . ~}$

Otros estudios han explorado aspectos de la funcionalidad familiar relacionados con la adherencia a tratamientos de pacientes con diabetes tipo 2 y con insuficiencia renal ${ }^{29-31}$, pero en ellos no se exploró su percepción de salud utilizando el SF-36, ni se definieron variables laborales con las que se pudiera comparar el presente estudio.

\section{Características y condiciones estudiadas que aportan a la explicación de la disfuncionalidad familiar}

En este estudio, explican la disfuncionalidad familiar de los vendedores el estado civil, siendo los casados y en unión libre, quienes menos perciben disfuncionalidad familiar en comparación con los solteros. De otro lado, quienes vivían en casa arrendada tenían mayor prevalencia de riesgo de disfuncionalidad familiar, y en cuanto a las condiciones laborales, aportan a la explicación haber sido ventero entre 16 y 40 años, y haber sido obrero antes de ser ventero. La mayor disfuncionalidad familiar desde las condiciones laborales exploradas fue explicada por la tipología de venta, específicamente para quienes vendían bebidas picadura y dulce, y con relación a la percepción de salud, explicaron una menor percepción de disfuncionalidad familiar tener un desempeño físico y emocional regular y malo, función social buena, regular y mala, y una vitalidad buena y regular, siendo estadísticamente significativa la capacidad explicativa del desempeño físico regular, y el dominio de salud mental. Estos dos últimos, son los que más explican la disfuncionalidad familiar.

\section{Conclusiones}

Las características que se asociaron y que más explican la disfuncionalidad familiar en esta población trabajadora fueron: recibir menos ingresos al mes, tener menos personas a cargo, no ser cabeza de familia, pertenecer a un estrato socio económico bajo, vender bebidas, picadura y dulce, percibir peor estado de salud en los dominios de dolor corporal, función social, salud general, salud mental y vitalidad. De otro lado, también se observó una tendencia de mayor disfuncionalidad familiar para quienes eran mayores y presentaron peor percepción de su función física.

Las características que más explican la disfuncionalidad familiar en términos de su asociación estadísticamente significativa fueron la tipología de venta (vender bebidas picadura y dulce), tener un regular desempeño físico y percepción de salud mental buena, regular o mala. Tendencias explicativas de disfuncionalidad familiar fueron presentadas por el estado civil (soltero), tener menos de 16 años de laborar como ventero, vivir en casa arrendada, percepción de desempeño emocional bueno y malo, desempeño físico bueno y regular, función social bueno, regular y mala, y vitalidad regular y buena.

En general, las características observadas son orientadoras de la vulnerabilidad laboral en este grupo, según los parámetros descritos por organismos de salud y trabajo internacionales y los reportes oficiales a nivel nacional ${ }^{4-6,8,31}$. Aunque en los estudios revisados aún no se incluyen este tipo de exploraciones, deberían incluirse, pues se dificulta cualquier tipo de comparación y a su vez el avance en el tema específico, en un continente donde el número considerable de trabajadores informales "vendedores" que hacen de las calles y aceras de las ciudades su lugar de trabajo, merece más atención desde la academia y los entes gubernamentales, entendiendo que no es una novedad que las condiciones en que viven $\mathrm{y}$ trabajan las personas determinan de manera importante el proceso de salud-enfermedad en los individuos, los grupos de personas y la sociedad en general ${ }^{8-10}$. Otros estudios de carácter analítico, tipo cohorte o de intervención, podrían evidenciar con datos de incidencias como a mayor vulnerabilidad laboral del ventero, es mayor la 
disfuncionalidad familiar o realizar intervenciones comunitarias para tratar de disminuir tal condición en este tipo de población trabajadora beneficiando a su familia y a la sociedad.

\section{Conflicto de intereses: Ninguno declarado por} los autores.

\section{Referencias}

1. Gallego H.A. Recuperación crítica de los conceptos de familia, dinámica familiar y sus características. [internet]. Revista Virtual Universidad Católica del Norte. No.35. Febrero - Mayo de 2012, ISSN 0124.- 5821. Pag. 326-45 [Consulta: 2015 mar 07]. Disponible en: http://revistavirtual.ucn.edu.co/index.php/RevistaUC N/article/viewFile/364/679

2. Palacios J, Rodrigo MJ. La familia como contexto de desarrollo humano. Familia y desarrollo Humano. Madrid. Alianza. 2001.

3. Minuchin S. Familias y terapia familiar Buenos Aires: Gedisa. Minuchin, S. Calidoscopio familiar. Barcelona: Paidós. 1984.

4. Organización Internacional del Trabajo - OIT. Oficina Regional para América Latina y el Caribe. Panorama laboral 2013 - América Latina y el Caribe. 20 años panorama laboral. Primera edición. ISSN: 2305-0276 (versión web pdf). 2013. 136 p. [intermnet]. [Consulta: 2014 sep. 04]. Disponible en: http://ilo.org/americas/publicaciones/panoramalaboral/WCMS_232760/lang--es/index.htm Organización Internacional del Trabajo - OIT. Informe anual sobre los derechos de los trabajadores - trabajar en el sector informal: una oportunidad, un riesgo, un reto. Dossier realizado para la CMT por la agencia InfoSud-Belgique; Capítulo 3: Condiciones de trabajo contrarias a las normas. Junio 2002.

5. Organización Panamericana de la Salud - OPS. Plan Regional en Salud de los Trabajadores. 57pg. [Internet]. [Consulta: 2015 ene 19]. Disponible en: http://www.who.int/occupational_health/regions/en/ oehamplanreg.pdf.

6. Pizarro R. La vulnerabilidad social y sus desafíos: Una mirada desde América Latina. Serie 6. Estudios estadísticos y prospectivos. División de Estadística y Proyecciones Económicas. Santiago de Chile, Naciones Unidas- CEPAL. Febrero de 2001: 71.

7. Organización Mundial de la Salud. Comisión sobre Determinantes Sociales de la Salud. Informe final, subsanar las necesidades en una generación. Alcanzar la equidad sanitaria actuando sobre los determinantes sociales de la salud. Washington. 2009.31pg [Internet]. [Consulta: 2015 ene 31]. Disponible en: http://whqlibdoc.who.int/publications/2009/978924 3563701_spa.pdf?ua=1

8. Giatti L, Barreto SM, César CC. Informal work, unemployment and health in Brazilian metropolitan areas, 1998 and 2003. [serial on the Internet]. Cad.
Saúde Pública. 2008;24(10): 2396-406. [cited 2015 Mar 07]; Disponible en: http://www.scielo.br/scielo.php?script=sci_arttext\&pi $\mathrm{d}=$ S0102-311X2008001000020\&lng=en. http://dx.doi.org/10.1590/S0102311X2008001000020.

9. Giatti L, Barreto SM, César CC. Household context and self-rated health: the effect of unemployment and informal work. JECH. [Internet]. Nunio de 2008; 62(2): 1079-85 [Consulta: 28 de agosto de 2008]. Disponible en: http://jech.bmj.com/content/62/2.toc

10. Asociación Latinoamericana de Medicina Social ALAMES. Taller Latinoamericano de Determinantes Sociales de la Salud. México septiembre -octubre de 2008. 155pg [Internet]. [Consulta: 2015 ene 31]. Disponible http://www.alames.org/documentos/ponencias.pdf

11. More R. Economía política de los determinantes sociales de la salud. Marco general y bases conceptuales para su aplicación preliminar a la realidad del Perú. Informe Final, Lima diciembre de 2009: 116. [Internet]. [Consulta: 2015 ene 29]. Disponible en: http://www.bvsde.paho.org/texcom/sct/048025.pdf

12. Garzón, DM. Condiciones de salud enfermedad de un grupo de trabajadores informales "venteros" del centro de Medellín. 2008-2009. 252pg. [Investigación para optar al título de Magister en Epidemiologia]. Medellín: Universidad de Antioquia - Facultad Nacional de Salud Pública; 2010.

13. García H, Lugo-Agudelo LE. Adaptación cultural y fiabilidad del cuestionario de calidad de vida en salud SF-36 en instituciones de salud de Medellín, Colombia 2002. Maestría en epidemiología con enfasis en epidemiología clínica. Medellín, Facultad Nacional de Salud Pública. Universidad de Antioquia. 2002. 75p.

14. Arías LC. Herrera JA. El APGAR familiar en el cuidado primario de salud. Universidad del Valle. Cali-Colombia. Revista Colombia Médica. 1994;25:26-08.

15. Suarez CM, Alcala EM. APGAR Familiar: una herramienta para detectar disfunción familiar [internet]. Rev. Méd. La Paz. Enero - Jun io 2014. v.20 n.1. 53-7 [Consulta: 2015 marzo 21]. Disponible en: http://www.scielo.org.bo/scielo.php?pid=S172689582 014000100010\&script=sci_arttex

16. Forero L, Avendaño MC, Duarte ZJ, Campo-Arias A. Consistencia interna y análisis de factores de la escala APGAR para evaluar el funcionamiento familiar en estudiantes de básica secundaria. [serial on the Internet]. rev.colomb.psiquiatr. 35(1): 23-29. 2006 Mar [cited 2015 Mar 21]; Available from: http://www.scielo.org.co/scielo.php?script=sci_arttext \&pid=S0034-74502006000100003\&lng=en

17. Arias C. Herrera JA. El APGAR familiar en el cuidado primario de salud. Colombia Medica. North America. [internet]. Enero - junio de 1994. Vol. 25: 26-38 [Consulta: 2015 marzo 07]. Disponible en: http://colombiamedica.univalle.edu.co/index.php/com edica/article/view/1776

18. Ortega AA, Fernandez VA, Osotio PM. Funcionamiento familiar su percepción por diferentes grupos generacionales y signos de alarma. [internet]. Rev 
Cubana Med Gen Integr. Agosto 2003. Vol 19(4) [Consulta: 2015 mar 07]. Disponible en: http://scielo.sld.cu/scielo.php?script=sci_arttext\&pid= S086421252003000400002\&lng=es

19. Giraldo CA, Morales-Giraldo LF, Gushiken- Miyagui A, et al. Funcionalidad familiar en los hogares comunitarios de Itagüí. [ internet]. Revista Pediatría, 1998. [Consulta: 2009 ag 16]. Disponible en: http://www.encolombia.com/pediatria3522000funcionalidad.htm

20. Guibert-Reyes W, Torres-Miranda N. Intento suicida y funcionamiento familiar. Rev Cubana Med Gen Integr [revista en la Internet]. [citado 2015 Mar 12] ; 17(5): 452-460. 2001 Oct. Disponible en: http://scielo.sld.cu/scielo.php?script=sci_arttext\&pid= S086421252001000500008\&lng=es

21. Colombia, Ministerio de la Protección Social - Fondo de Riesgos Profesionales. Diagnóstico Nacional de condiciones de salud y trabajo de las personas ocupadas en el sectror informal de la economía de 20 departamentos de Colombia y propuesta de monitoreo de estas condiciones. ISBN: 978-958-8361-56-7. Medellín; Agosto de 1998. 142p.

22. Colombia. Ministerio de Hacienda y Crédito Público Dirección General de Política Macroeconómica. Centro de Estudios Fiscales. Bogotá. D.C, Colombia. [internet]. ISSN 2027-8365. Agosto 09 de 2011. 36 pg. [Consulta: 2015 marzo 16]. Disponible en: http://www.minhacienda.gov.co/MinHacienda/hacien dapublica/CentroEstudios/publicaciones/notas

23. Colombia. Ministerio de Trabajo. Organización Iberoaméricana de Seguridad Social (OISS). Segunda encuesta nacional de condiciones de seguridad y salud en el trabajo en el Sistema General de Riesgos Laborales de Colombia. Bogotá D.C., diciembre de 2013. 56pag. [intermnet]. [Consulta: 2014 sep. 02]. Disponible en: http://ccs.org.co/salaprensa/images/Documentos/INF ORME_EJECUTIVO_II\%20ENCSST.pdf

24. García A, Santibanez M, Soriano G. Utilización de un cuestionario de salud percibida (SF-36) en vigilancia de la salud de los trabajadores Revista Riesgos laborales España. 2004;7(3):88-98.

25. Zabala GM, Dominguez SG. Depresión y percepción de la funcionalidad familiar en adultos mayores urbanos mexicanos. Psicogeriatría [internet]. Marzo de 2010; 2 (1): 41-48 [Consultado: 2015 marzo 18]. Disponible en: http://www.psiquiatria.com/depresion/depresion-ypercepcion-de-la-funcionalidad-familiar-en-adultosmayores-urbanos-mexicanos/

26. Ortega-Alvelay A, Fernandez-Vidad AT, Osotio-Pagola MF. Funcionamiento familiar $\mathrm{Su}$ percepción por diferentes grupos generacionales y signos de alarma Revista Cubana de Medicina General Integral. 2003:19(4).

27. Trujillo BW, Róman HJ, Lombard HA, Remoir FE, Arredondo NO, Martinez PE, et al. Adaptación del cuestionario SF-36 para medir calidad de vida relacionada con la salud en trabajadores Cubanos. Revista Cubana de Salud y Trabajo [internet]. 2014;15(1):62-70. [Consultado 2015 marzo 16]. Disponible http://www.medigraphic.com/pdfs/revcubsaltra/cst2014/cst141j.pdf

28. Rodriguez AG, Rodriguez AI. Instituto Mexicano del Seguro Social. Disfunción familiar en pacientes con insuficiencia renal crónica. [internet]. Rev Med IMSS 2004; 42(2):97-102 [Consulta: 2015 marzo 22]. Disponible http://www.medigraphic.com/pdfs/imss/im2004/im042b.pdf

29. Mendez LD, Gómez LV, García RM, Perez LJ, Avarrete EA. Instituto Mexicano del Seguro Social. Disfunción familiar y control del paciente diabético tipo 2. [internet]. Rev Med IMSS 2004; 42 (4): 281-284 [Consulta: 2015 marzo 22]. Disponible en: http://www.medigraphic.com/pdfs/imss/im2004/im044b.pdf

30. Pizarro R. La vulnerabilidad social y sus desafios: una mirada desde América Latina. Serie 6. Estudios estadísticos y prospectivos. División de Estadística y Proyecciones Económicas. Santiago de Chile, Naciones Unidas- CEPAL. Febrero de 2001.71 pag [Internet]. [Consulta: 2015 mar 01]. Disponible en: http://repositorio.cepal.org/bitstream/handle/11362/ 4762/S0102116_es.pdf?sequence=1 